Supporting Information

\title{
Improving the Activity of DNA-Encoded Sensing Elements Through Confinement in Silk Microcapsules
}

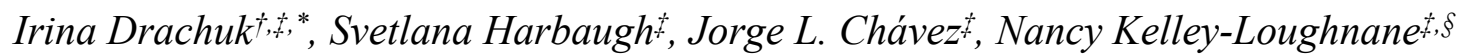 \\ $\dagger$ UES Inc., Dayton, OH 45432
}

$\$ 711^{\text {th }}$ Human Performance Wing, Airmen Systems Directorate, Air Force Research Laboratory, Wright-Patterson AFB, OH 45433

$\S$ Materials and Manufacturing Directorate, Air Force Research Laboratory, Wright-Patterson AFB, OH 45433

*Corresponding author email: irina.drachuk.ctr@us.af.mil

A

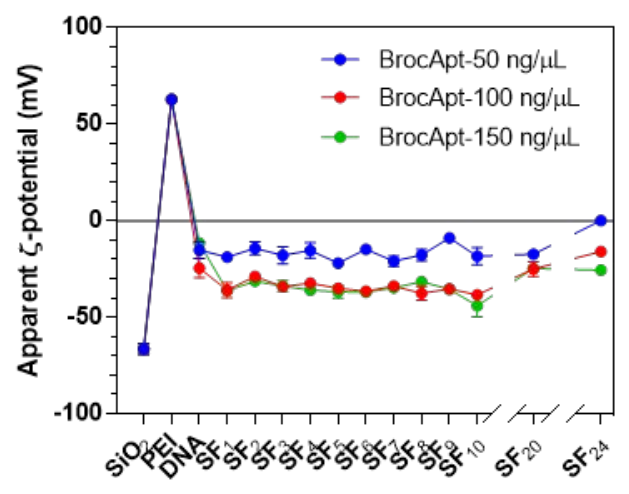

B

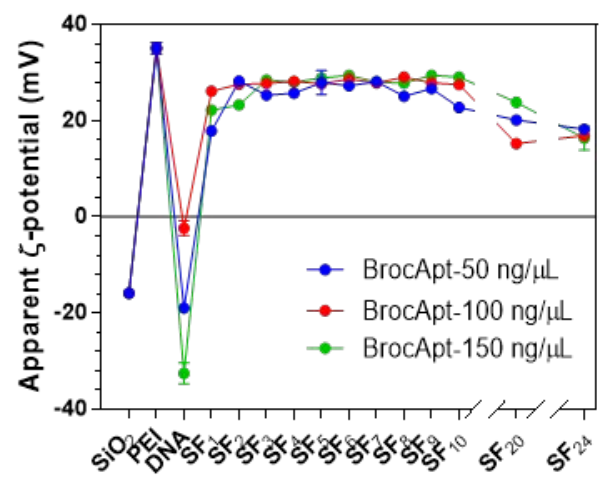

Figure S1. Zeta potential for (SF) capsules immobilized with different concentration of DNA plasmids encoding BrocApt. Adsorption was performed on $\mathrm{SiO}_{2}$ cores functionalized with PEI layer. Concentration of PEI was $6 \mathrm{mg} / \mathrm{mL}$ (A) and $0.5 \mathrm{mg} / \mathrm{mL}$ (B). 

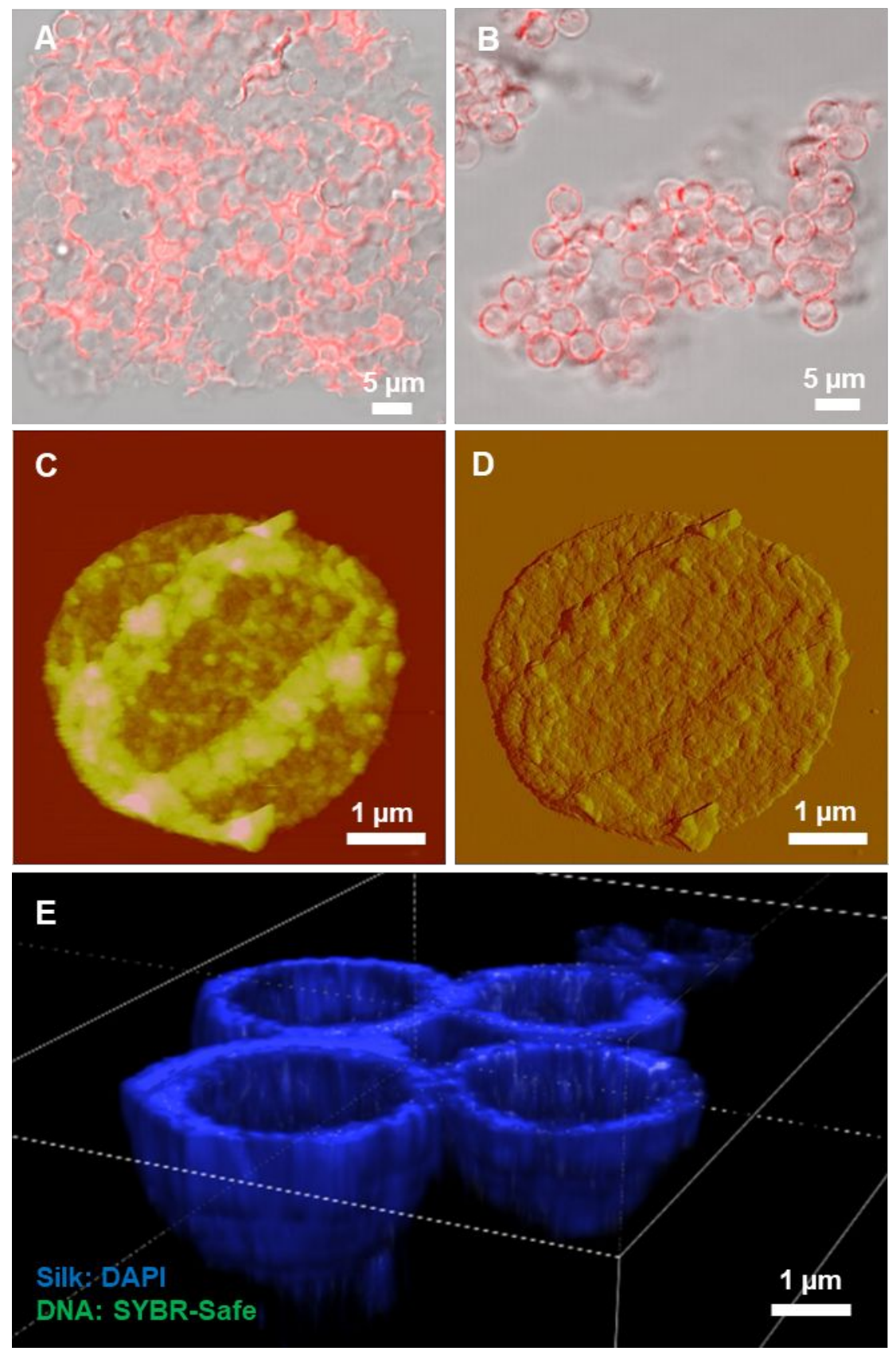

Figure S2. Characterization of SF capsules. (A, B) CLSM images of (SF) ${ }_{14}$ microcapsules prepared with no PEI prime layer (A) and $0.5 \mathrm{mg} / \mathrm{mL}$ PEI layer (B) prior to adsorption of DNA plasmids on $\mathrm{SiO}_{2}$ cores. (C, D) AFM height and amplitude error images of control (no DNA) (SF) ${ }_{20}$ capsules. Z-scale is $400 \mathrm{~nm}$ for C, and $500 \mathrm{mV}$ for D. (E) CLSM image of control (no DNA) $(\mathrm{SF})_{20}$ capsules stained with SYBR-Safe stain confirming no non-specific labeling of the silk fibroin. 
Due to selective permeability of the capsule membrane to the molecules of a certain size, capsules porosity can be estimated. Figure S3 shows representative images of (SF) capsules that were considered to be completely permeable (outside and inside intensities were comparable), partially permeable (50-70\% of capsules were not permeable) and completely not permeable (inside fluorophore intensity was lower compared to outside intensity). Analysis of the fluorescent images for (SF) capsules with variable number of layers prepared with $6 \mathrm{mg} / \mathrm{mL}$ of PEI allowed to estimate MWCO to be $\sim 23 \mathrm{~nm}$ (based on the measured hydrodynamic radii for $500 \mathrm{kDa}$ (20 $\mathrm{nm})$ and $2 \mathrm{MDa}(25 \mathrm{~nm})$ dextran molecules). However, the porosity of $(\mathrm{SF})_{14}$ capsules prepared with $0.5 \mathrm{mg} / \mathrm{mL}$ of PEI was estimated to be $9 \mathrm{~nm}$ (Table S1).

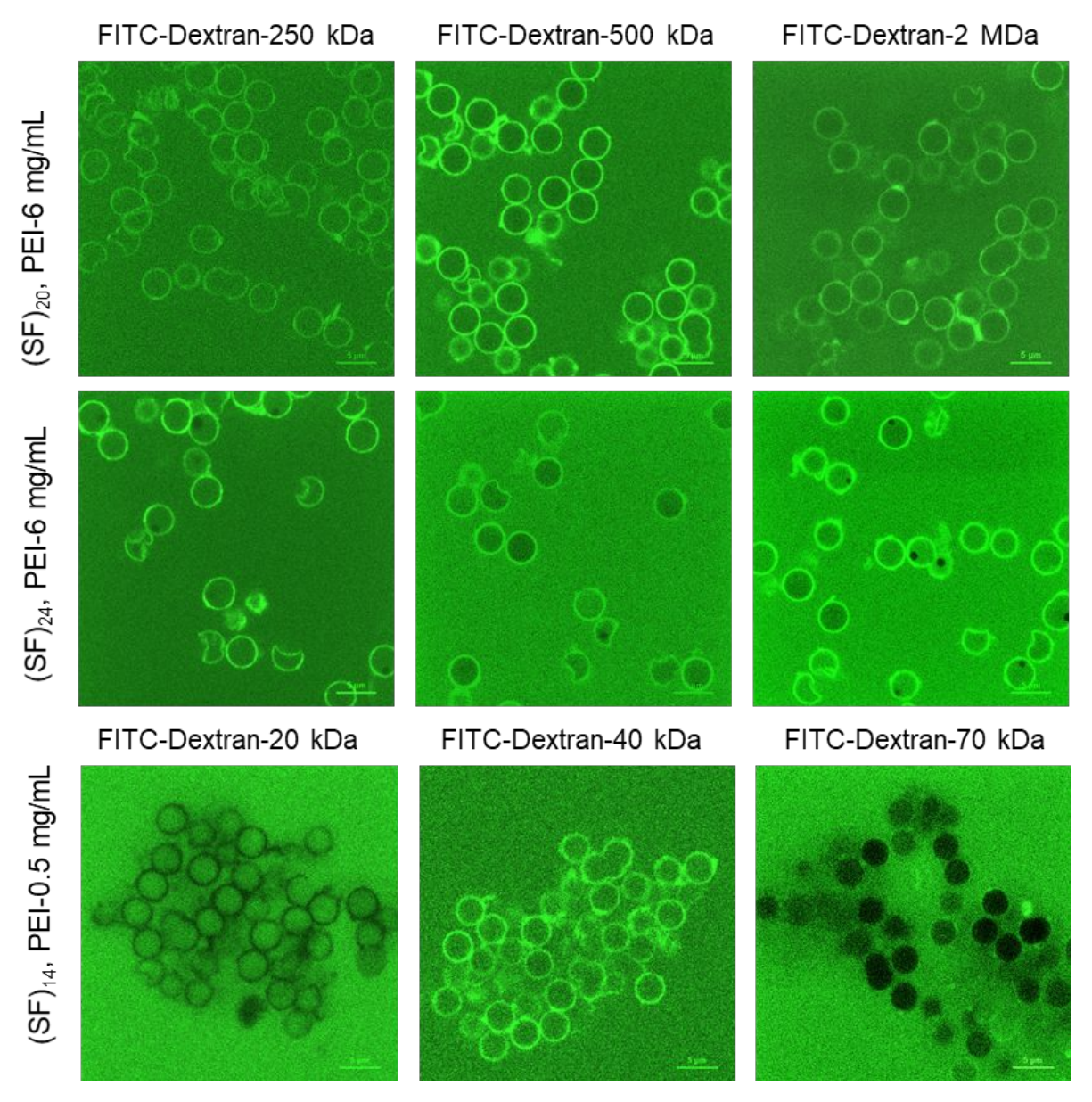

Figure S3. CLSM representative fluorescent images of $(\mathrm{SF})_{n}$ microcapsules prepared with different PEI concentrations after incubation in FITC-Dextran solutions of various $\mathrm{Mw}\left(20 \mu \mathrm{M}, \mathrm{H}_{2} \mathrm{O}\right)$. 
Table S1. Porosity of $(\mathrm{SF})_{n}$ microcapsules prepared using various combinations of contributing factors.

\begin{tabular}{|c|c|c|c|}
\hline $\begin{array}{c}\text { Number of } \\
\text { Layers }\end{array}$ & $\begin{array}{c}\text { PEI } \\
\text { Concentration }\end{array}$ & $\begin{array}{c}\text { Concentration of } \\
\text { SF }\end{array}$ & Porosity \\
\hline 10 & $6 \mathrm{mg} / \mathrm{mL}$ & $0.6 \mathrm{mg} / \mathrm{mL}$ & $26 \mathrm{~nm}$ \\
\hline 14 & $6 \mathrm{mg} / \mathrm{mL}$ & $0.6 \mathrm{mg} / \mathrm{mL}$ & $25 \mathrm{~nm}$ \\
\hline 16 & $6 \mathrm{mg} / \mathrm{mL}$ & $1 \mathrm{mg} / \mathrm{mL}$ & $24 \mathrm{~nm}$ \\
\hline 20 & $6 \mathrm{mg} / \mathrm{mL}$ & $1 \mathrm{mg} / \mathrm{mL}$ & $25 \mathrm{~nm}$ \\
\hline 24 & $6 \mathrm{mg} / \mathrm{mL}$ & $1 \mathrm{mg} / \mathrm{mL}$ & $23 \mathrm{~nm}$ \\
\hline 10 & $0.5 \mathrm{mg} / \mathrm{mL}$ & $0.6 \mathrm{mg} / \mathrm{mL}$ & $10 \mathrm{~nm}$ \\
\hline 14 & $0.5 \mathrm{mg} / \mathrm{mL}$ & $0.6 \mathrm{mg} / \mathrm{mL}$ & $9 \mathrm{~nm}$ \\
\hline
\end{tabular}




\section{Calculations for Amount of DNA loaded in the microcapsule.}

The mass of DNA $\left(m_{i}\right)$ loaded in each capsule was calculated based on the following:

$$
m_{i}=\frac{C \times V}{N}
$$

where $C$ - concentration of plasmid DNA,

$V$ - volume,

$N$ - number of $\mathrm{SiO}_{2}$ cores used for initial encapsulation.

Number of cores used for DNA adsorption was calculated based on a calibration curve obtained from serial dilution $(1: 10,1: 50 ; 1: 100 ; 1: 500 ; 1: 1000)$ of silica beads standards of comparable size $(4 \mu \mathrm{m})$ (Figure $\mathrm{S} 4)$. The absorbance of $\mathrm{SiO}_{2}$ cores $\left(\mathrm{A}_{320}=2.36\right)$ was used for calculating the number of cores for DNA encapsulation. Logarithmic curve fitting allowed to estimate the number of cores, $N=6.769 \times 10^{11}$. This data was used to approximate the mass and number of DNA copies loaded in each bioreactor.

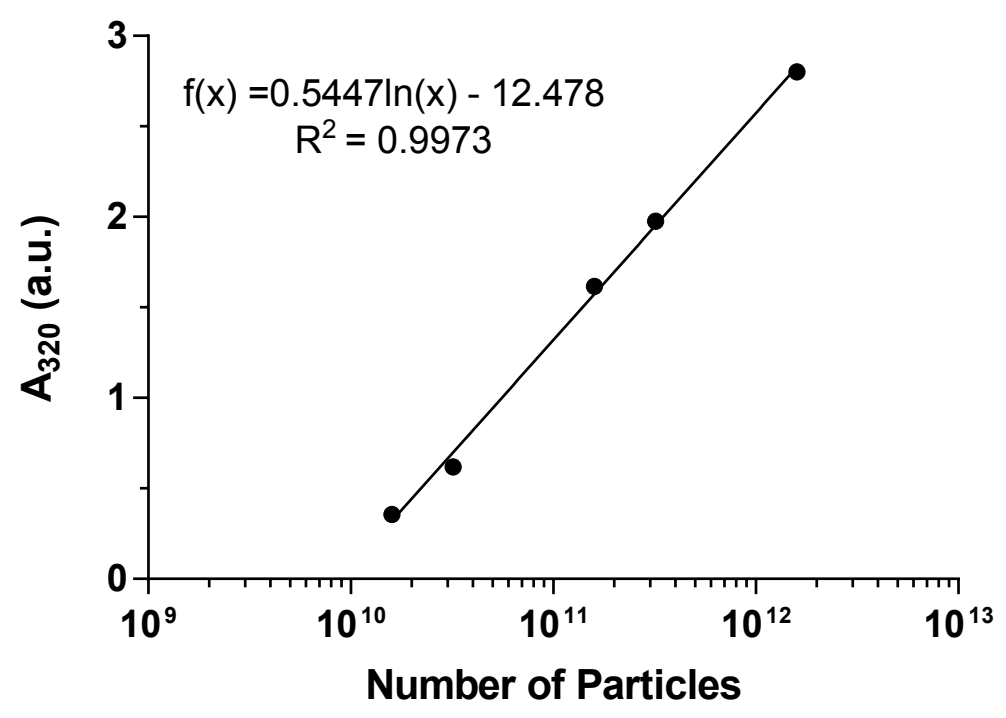

Figure S4. Calibration curve for $\mathrm{SiO}_{2}$ particles. 
Calculations of mass $\left(m_{i}\right)$ of DNA loaded in each core/capsule.

Assuming that $80 \%$ of DNA retained after encapsulation, $m_{i}$ was calculated accordingly:

$$
m_{i}=\frac{C \times V}{N} \times 0.8
$$

1) For $50 \mathrm{ng} / \mu \mathrm{L}$ initial DNA concentration:

$$
\begin{aligned}
m_{i}=\frac{C \times V}{N} & \times 0.8=\frac{50 \times 10^{-6}\left(\frac{g}{m L}\right) \times 1(m L)}{6.769 \times 10^{11}(\text { cores })} \times 0.8=\frac{50 \times 10^{-6}(\mathrm{~g})}{6.769 \times 10^{11}(\text { cores })} \times 0.8=5.91 \\
& \times 10^{-17}\left(\frac{g}{\text { core }}\right)=0.059(\mathrm{fg} / \text { capsule })
\end{aligned}
$$

2) For $100 \mathrm{ng} / \mu \mathrm{L}$ initial DNA concentration:

$m_{i}$

$$
\begin{aligned}
& =\frac{C \times V}{N} \times 0.8=\frac{100 \times 10^{-6}\left(\frac{g}{m L}\right) \times 1(\mathrm{~mL})}{6.769 \times 10^{11} \text { cores }} \times 0.8=\frac{100 \times 10^{-6}(\mathrm{~g})}{6.769 \times 10^{11}(\text { cores })} \times 0.8=11.8 \\
& \times 10^{-17}\left(\frac{\mathrm{g}}{\text { core }}\right)=0.118(\mathrm{fg} / \text { capsule })
\end{aligned}
$$

3) For $150 \mathrm{ng} / \mu \mathrm{L}$ initial DNA concentration:

$$
\begin{aligned}
m_{i}=\frac{C \times V}{N} & \times 0.8=\frac{150 \times 10^{-6}\left(\frac{g}{m L}\right) \times 1(m L)}{6.769 \times 10^{11}(\text { cores })} \times 0.8=\frac{150 \times 10^{-6}(g)}{6.769 \times 10^{9}(\text { cores })} \times 0.8=17.73 \\
& \times 10^{-17}\left(\frac{g}{\text { core }}\right)=0.177(\text { fg/capsule })
\end{aligned}
$$

Number of ThyRS plasmid DNA copies immobilized in each core/capsule $\left(N_{\text {ThyRS }}\right)$ :

$$
N_{\text {ThyRS }}=\frac{m_{i}}{M_{w}} \times N_{A}
$$

1) For $50 \mathrm{ng} / \mu \mathrm{L}$ initial loading concentration:

$$
N_{\text {ThyRS }}=\frac{m_{i}}{M_{w}} \times 6.022 \times 10^{23}=\frac{0.059 \times 10^{-15} \mathrm{~g}}{2.22 \times 10^{6} \mathrm{~g} / \mathrm{mol}} \times 6.022 \times 10^{23}=16(\text { DNA } / \text { capsule })
$$

2) For $100 \mathrm{ng} / \mu \mathrm{L}$ initial loading concentration: 


$$
N_{\text {ThyRS }}=\frac{m_{i}}{M_{w}} \times 6.022 \times 10^{23}=\frac{0.118 \times 10^{-15} \mathrm{~g}}{2.22 \times 10^{6} \mathrm{~g} / \mathrm{mol}} \times 6.022 \times 10^{23}=32(\text { DNA } / \text { capsule })
$$

3) For $150 \mathrm{ng} / \mu \mathrm{L}$ initial loading concentration:

$$
N_{\text {ThyRS }}=\frac{m_{i}}{M_{w}} \times 6.022 \times 10^{23}=\frac{0.177 \times 10^{-15} \mathrm{~g}}{2.22 \times 10^{6} \mathrm{~g} / \mathrm{mol}} \times 6.022 \times 10^{23}=48(\text { DNA } / \text { capsule })
$$

Number of BrocApt. plasmid DNA copies immobilized in one capsule $\left(N_{\text {BrocApt }}\right)$ :

$$
N_{\text {BrocApt }}=\frac{m_{i}}{M_{w}} \times N_{A}
$$

1) For $50 \mathrm{ng} / \mu \mathrm{L}$ initial loading concentration:

$$
N_{\text {BrocApt }}=\frac{m_{i}}{M_{w}} \times 6.022 \times 10^{23}=\frac{0.059 \times 10^{-15} \mathrm{~g}}{3.54 \times 10^{6} \mathrm{~g} / \mathrm{mol}} \times 6.022 \times 10^{23} \approx 10(\text { DNA/capsule })
$$

2) For $100 \mathrm{ng} / \mu \mathrm{L}$ initial loading concentration:

$$
N_{\text {BrocApt }}=\frac{m_{i}}{M_{w}} \times 6.022 \times 10^{23}=\frac{0.118 \times 10^{-15} \mathrm{~g}}{3.54 \times 10^{6} \mathrm{~g} / \mathrm{mol}} \times 6.022 \times 10^{23} \approx 20(\text { DNA/capsule })
$$

3) For $150 \mathrm{ng} / \mu \mathrm{L}$ initial loading concentration:

$$
N_{\text {BrocApt }}=\frac{m_{i}}{M_{w}} \times 6.022 \times 10^{23}=\frac{0.177 \times 10^{-15} \mathrm{~g}}{3.54 \times 10^{6} \mathrm{~g} / \mathrm{mol}} \times 6.022 \times 10^{23} \approx 30(\text { DNA/capsule })
$$




\section{Calculations of concentration of encapsulated DNA in cell-free reaction.}

The concentration of encapsulated DNA was calculated based on the number of microcapsules added to the reaction (Rxn.), and was derived by measuring absorbance at $320 \mathrm{~nm}$ $\left(\mathrm{A}_{320}\right)$ using the calibration curve obtained for $\mathrm{SiO}_{2}$ standards (Figure S5). Table S2 summarizes the data calculations for encapsulated DNA for each of the capsule type used in the cell-free reaction $(50 \mu \mathrm{L})$.

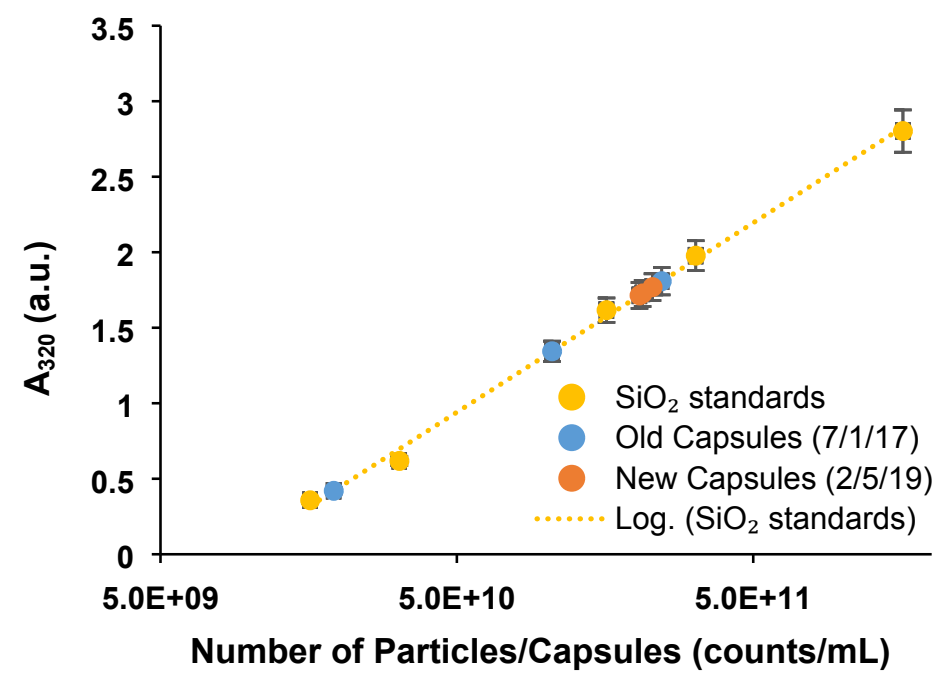

Figure S5. Calibration curve for $\mathrm{SiO}_{2}$ standards and absorbance measurements for freshlyprepared and old $(\mathrm{SF})_{20}$ microcapsules. 
Table S2. Concentration of Encapsulated DNA in Cell-Free Reactions.

\begin{tabular}{|c|c|c|c|c|}
\hline $\begin{array}{c}\text { Type of } \\
\text { Capsule }\end{array}$ & $\mathbf{A}_{\mathbf{3 2 0}}$ & $\begin{array}{c}\text { Number of } \\
\text { Capsules in 1 mL }\end{array}$ & $\begin{array}{c}\text { Mass of DNA per } \\
\text { Rxn. }\end{array}$ & $\begin{array}{c}\text { Concentration } \\
\text { of DNA in Rxn. } \\
(\mathbf{n g} / \boldsymbol{\mu L})\end{array}$ \\
\hline $\begin{array}{c}(\mathrm{SF})_{20^{-}} 50 \text { (old } \\
\text { capsules) }\end{array}$ & 0.42 & $1.921 \times 10^{11}$ & $2.041 \times 10^{-8}$ & 0.4 \\
\hline $\begin{array}{c}(\mathrm{SF})_{20^{-}}-100 \text { (old } \\
\text { capsules) }\end{array}$ & 1.344 & $1.0481 \times 10^{11}$ & $5.57 \times 10^{-8}$ & 1.1 \\
\hline $\begin{array}{c}(\mathrm{SF})_{20^{-}}-150 \text { (old } \\
\text { capsules) }\end{array}$ & 1.808 & $2.456 \times 10^{11}$ & $3.914 \times 10^{-7}$ & 7.5 \\
\hline $\begin{array}{c}(\mathrm{SF})_{20}-50 \text { (new } \\
\text { capsules) }\end{array}$ & 1.769 & $2.286 \times 10^{11}$ & $1.2164 \times 10^{-7}$ & 2.5 \\
\hline $\begin{array}{c}(\mathrm{SF})_{20}-100 \\
(\text { new capsules) }\end{array}$ & 1.727 & $2.1172 \times 10^{11}$ & $2.2485 \times 10^{-7}$ & 4.5 \\
\hline $\begin{array}{c}(\mathrm{SF})_{20}-150 \\
(\mathrm{new} \mathrm{capsules})\end{array}$ & 1.714 & $2.0673 \times 10^{11}$ & $3.293 \times 10^{-7}$ & 6.6 \\
\hline
\end{tabular}

A

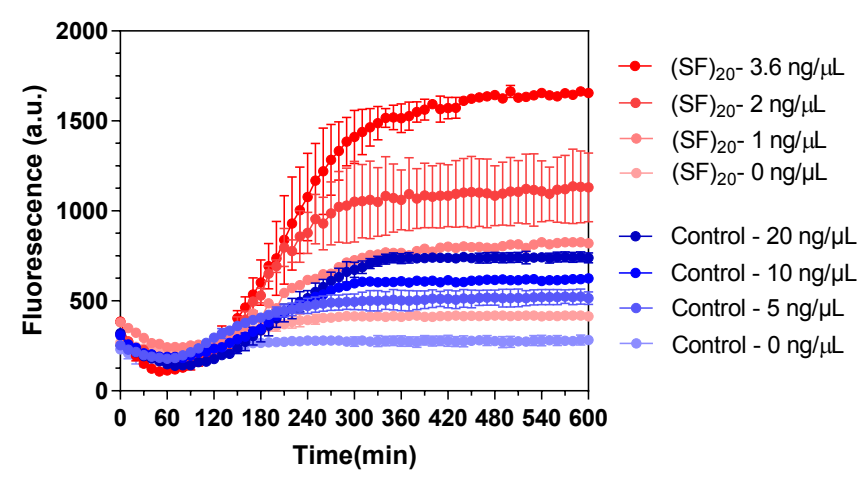

B

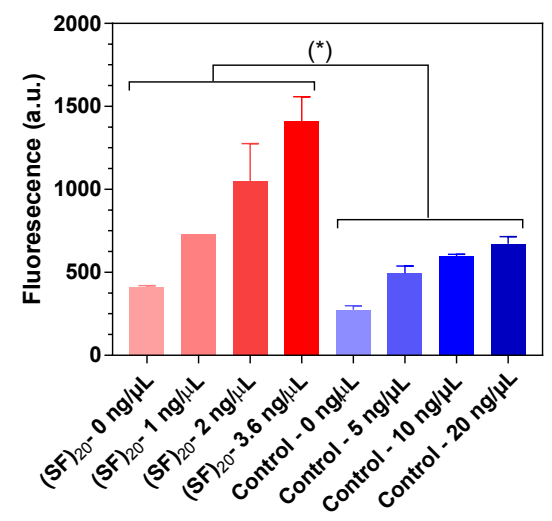

Figure S6. Comparison of BroccApt activation from DNA immobilized in (SF) ${ }_{20}$ capsules (red) and control DNA (blue) after long-term storage. (A) Kinetics of BrocApt activation with DHBFI$1 \mathrm{~T}\left(100 \mu \mathrm{M}, \mathrm{diH}_{2} \mathrm{O}\right)$ during incubation in cell-free system (PURExtract, NE BioLabs). (B) Endpoint data after $5 \mathrm{hr}$ of incubation. Control DNA was stored at $-20^{\circ} \mathrm{C}$ for one year, and $(\mathrm{SF})_{20}$ capsules were stored as aqueous solutions at ambient conditions for 2 years. $P<0.01(\mathrm{n}=3)$. 

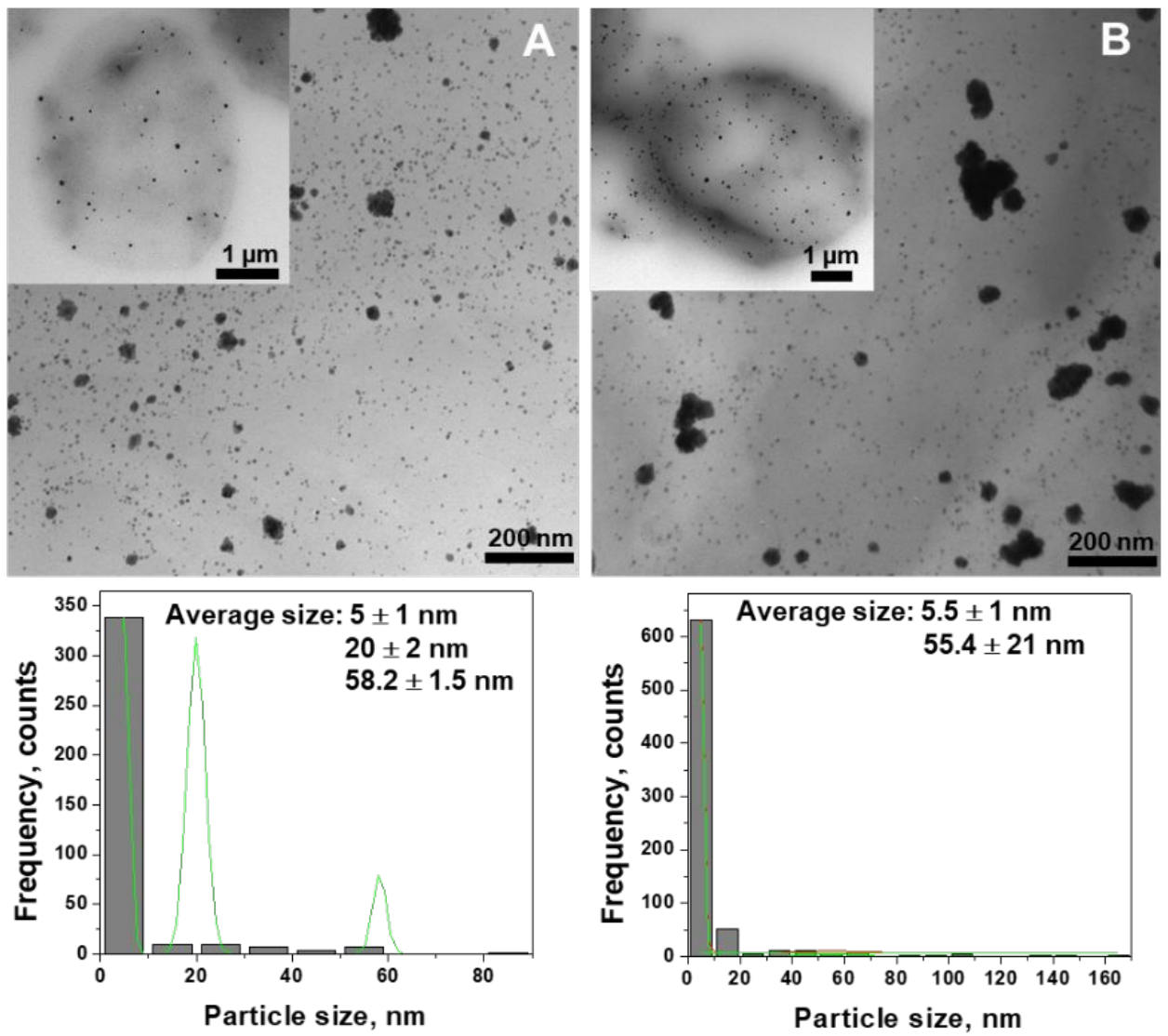

Figure S7. TEM images and corresponding size distribution profiles for AuNPs in $(\mathrm{SF})_{16}$ capsules after incubation in $1 \mathrm{mM}(\mathrm{A})$ and $2 \mathrm{mM} \mathrm{HAuCl}_{4}$ (B). The insets shows individual $(\mathrm{SF})_{16}$ microcapsules. 